EPiC Series in Engineering
Volume 3, 2018, Pages 2204-2215
HIC 2018. 13th International
Conference on Hydroinformatics

\title{
Emerging pollutants in developing countries: optimizing sustainable treatment solutions
}

\author{
Zara Visanji ${ }^{1 *}$, Seyed M. K Sadr ${ }^{1}$, Matthew B.Johns ${ }^{1}$, Dragan Savic ${ }^{1}$ and \\ Fayyaz A. Memon ${ }^{1}$ \\ ${ }^{1}$ Centre for Water Systems, College of Engineering, Mathematics and Physical Sciences, Harrison \\ Building, North Park Road, University of Exeter, Exeter, Devon, EX4 4QF, United Kingdom.
}

\begin{abstract}
Over the past decade the research surrounding the occurrence, source, fate and removal of emerging pollutants has been increasing. The aim of this study was to create an add-on program which analyses the removal of emerging pollutants, to an existing decision support tool (WiSDOM). The tool was also used to evaluate the performance of each optimal solution in terms of removal of conventional pollutants using Multi Objective Genetic Algorithms and Multi Criteria Decision Analysis. Information was collated regarding minimum and maximum concentrations of emerging pollutants for surface water, groundwater, untreated wastewater, drinking water and treated wastewater. This information was used to populate an Excel Spreadsheet Program (ESP) which analysed the removal efficiencies of 13 different emerging pollutants for 42 wastewater treatment unit processes. The ESP is incorporated into the WiSDOM tool to allow the tool to calculate the removal of emerging pollutants. Three main scenarios were created to test the application of the tool and ESP. Scenario 1 focussed on the removal of emerging pollutants from from areas effected by tourism at different scales. Scenario 2 looked at the treatment suited for the removal of emerging pollutants from different socioeconomic regions. Lastly, Scenario 3 looked at removing emerging pollutants from hospital and industrial wastewater. The scenarios were focused on wastewater treatment in India and investigated the removal of 13 emerging pollutants commonly found in India.
\end{abstract}

\section{Introduction}

Emerging pollutants (EPs) can be defined as naturally occurring, synthetic or anthropogenic chemicals/substances or any microorganisms that are not regularly monitored. These substances are seen to have a negative impact on the environment and human health [1]. The most common classed EPs studied and discussed in the literature are pharmaceuticals, personal care products (PCPs) and endocrine disrupting chemicals (EDCs) [2]. Other EPs researched include: steroid hormones, surfactants, perfluorinated compounds (PFCs), flame retardants, industrial additives and agents, 
Emerging Pollutants in Developing Countries: Optimising Sustainable Treatment ... Z. Visanji et al.

gasoline additives, illicit drugs, UV filters (used in sunscreen products, cosmetics and creams) [3] and nanomaterials [4], [5]. EDCs are a cause for concern as they are seen to have an effect in the endocrine systems of aquatic animals, animals and humans. The effects to humans can result in changes to the reproductive health systems, birth defects, cancer issues and decreased male fertility [6]. Detergents, plastic bottles, flame retardants, food, toys, cosmetics, pesticides and steroids are included as EDCs due to their estrogenic activity [7]. Since 1965, there has been an increase in publications regarding pharmaceuticals and hormones in the water due to a noticed rise and increase in water pollution as a worldwide problem [8]. The problem with EPs is that they are not regularly monitored due to a lack of controlling requirements, legislations, and high analytical costs [9]. EPs are presented at low trace concentrations ranging from a few $\mathrm{ng} / \mathrm{L}$ to several $\mu \mathrm{g} / \mathrm{L}$, with point source locations consisting of industrial effluents, wastewater and water treatment plants. Historically these substances were not considered as pollutants, therefore treatment plants were not designed to remove them; resulting in EPs being able to enter freshwater and drinking water systems [10].

There has been an absence of research by governments and environmental organisations on EPs in developing countries before 2005; mainly due to inadequate funding for equipment, detection and quantification of EPs [11]. Since 2005, there has been a rise in research and publications regarding EPs in the aquatic environments in developing countries. However, there is still a lack of quantity of published research to allow for collaboration of data to identify the key areas of concern. Research has been carried out in developing countries focussing on the lack of removal of pollutants produced by agriculture and the textile industry [12]. Projects have been set up in some countries (Africa, Asia, Latin America and the Middle East) to allow for monitoring processes to be put in place [13], [14]. India, currently has no official legislations, however over 40 papers have been published including review papers highlighting the fate of EPs within India [15].

This paper describes the development and application of an Excel Spreadsheet Program (as an addin for a stand-alone user-friendly decision support tool called WiSDOM: WaStewater Decision support OptiMiser) used to calculate the removal rates of EPs during different treatment processes. Scenario examples based in India are used to demonstrate its application when combined with an existing decision support tool.

\section{Methodology}

This section looks at the methodology employed to analyse the removal of EPs from different treatment processes. Section 2.1 describes the scenarios developed and considered in this study for India and applied to the WiSDOM and ESP to test their application. All scenarios were defined in the context of India. Section 2.2 describes a decision support tool (WiSDOM) which was used in conjunction with the ESP. WiSDOM calculates the removal of conventional pollutants (Chemical oxygen demand (COD), biochemical oxygen demand (BOD), suspended solids (SS), total nitrogen (TN), phosphorus, faecal-Coliform, turbidity, intestinal nematode eggs and E-coli) from wastewater in India. Section 2.3 outlines the methodology which was used for the development of an Excel Spreadsheet Program (ESP), which was used to calculate the removal of thirteen EPs for forty-two treatment unit processes. Due to the limited published data on the removal of EPs in India, removal rates for different treatment unit processes were taken from a worldwide search to ensure a complete dataset. 


\subsection{Development of Scenarios within India}

Unlike the developed world, India currently has no official legislation or policy in place that specifically monitors the fate or management of EPs. India is currently one of the top pharmaceutical emerging markets in the world, and one of the largest global providers of drugs accounting for $20 \%$ of global exports. Proper waste management techniques do not exist in India, and conventional treatment plants are inefficient at the removal of EPs with sewage treatment plants discharging their effluent to rivers. The Bureau of Indian Standards are not currently addressing EPs [1]; therefore, it has become essential for the creation of baseline data to act as a framework for any future research or regulatory initiatives [16].

For this study 13 EPs were chosen which occurred mainly within India [15] but also appeared within the lists found within other countries or policies. The pollutants included in the study were: Amoxicillin (AMX), Bisphenol A (BPA), Carbamazepine (CBZ), Ciprofloxacin (CIP), Dichlorodiphenyltrichloroethane (DDT), Diclofenac (DCF), Dimethyl Phthalate (DMP), Endosulfan (END), Naproxen (NPX), Nonylphenol (NP), Norfloxacin (NOR), Ofloxacin (OFL), and Triclosan (TCS). The WiSDOM tool and ESP were run together to calculate the removal of the chosen EPs, by testing different theoretical scenarios based in India; to test the functionality of the ESP and its suitability as an addition to WiSDOM. These scenarios are described below.

Scenario 1, looked at investigating suitable treatment technologies which were able to remove EPs from areas effected by tourism at different scales: (A) Determining treatment options for areas that consist of 'Occasional Events' such as Diwali and Ganesh Chaturthi. "Occasional Events" can include festivals, public holidays and major sporting events [17]. (B) Determining treatment options for areas in India such as 'The Golden Triangle'. Both scenarios have known high tourism levels resulting in an expected increase of personal care products, medicine and illicit drugs. Table 1 presents the data for Scenarios 1(A) and (B) inputted into the WiSDOM tool, demonstrating the differences between Scenarios.

\begin{tabular}{lcc}
\hline \multicolumn{1}{c}{ Inputting Factors for WiSDOM } & Scenario 1(A) & Scenario 1(B) \\
\hline City/Town/Village & Panaji & Jaipur \\
State/Region & Goa & Rajasthan \\
Population to be served & 40,017 & $3,046,163$ \\
Wastewater produced (litres/person/day) & 150 & 150 \\
Average Income (INR/person/month) & 10,000 & 31,363 \\
Land Price (INR/Square metre) & 57,917 & 43,377 \\
Land Available for Treatment Plant (Square metre) & 52,000 & 10,000 \\
Budget available for capital costs (INR) & $700,000,000$ & $700,000,000$ \\
Budget available for annual O \& M costs (INR/year) & 500,000 & 500,000 \\
Intended use of effluent & Toilet Flushing & Toilet Flushing \\
Is the electricity source reliable & Yes & Yes \\
\hline
\end{tabular}

Table 1: Data used for Scenarios 1(A) and (B) inputted into the WiSDOM tool

Scenario 2, looked at treatment technologies which were suited to removing EPs from different socio-economic groups. (A) Determining treatment options for the removal of EPs in lower class 'slum' areas of India (Dahravi). (B) Determining treatment options for the removal of EPs in middle-upper class areas of India (Parel). (C) Determining treatment options for the removal of EPs in upper-class areas (Bandra). The diverse socio-economic groups will contain different treatment options for the removal of EPs, due to limitations regarding land availability and cost constraints. Therefore, it would be expected that the effluent of the 'slum' areas will contain a higher concentration of EPs, in comparison to the more affluent upper-class areas. Scenario 3, looked at treatment technologies suited 
Emerging Pollutants in Developing Countries: Optimising Sustainable Treatment ... Z. Visanji et al.

to the removal of EPs from different working environments. (A) Determining treatment options for the removal of EPs from Hospital wastewater (Ujjain). (B) Determining treatment options for the removal of EPs from Industrial wastewater (Perundurai).

\subsection{WiSDOM: An Existing Decision Support Tool}

WaStewater Decision support OptiMiser (WiSDOM) is a user-friendly tool designed to aid in the formulation of wastewater treatment trains for the removal of conventional pollutants in India. The decision support tool WiSDOM, was chosen as it determines the optimal treatment train options considering sustainability indicators and ensuring that the removal of conventional pollutants meet the Indian Water Quality Standards. At the core of the software, there is a technology library that contains detailed information on a wide range of wastewater treatment processes applicable within the context of India. The tool uses the technology library and Multi Objective Optimisation (MOO) algorithm to generate optimal wastewater treatment trains, which are then processed by a Multi-Criteria Decision Analysis (MCDA) technique to narrow down the resultant non-dominated solution set. There are two choices of MOO algorithm available to the user, the Non-Dominated Sorting Genetic Algorithm II (NSGAii) and the Omni-optimizer (Omni). The two algorithms have shown to handle the vagaries of practical optimisation problems well and prove suited to the formulation of wastewater treatment trains. The user has full control over the formulation of the problem; from defining which objectives are being considered for optimisation to the hydraulic, water quality, and design constraints. The available optimisation objectives are as follows, Capital Expenditure (CAPEX), Operational and Maintenance Expenditure (OPEX), Energy consumption, Sludge production, Land requirement, and Labour requirement, all of which are minimised by the optimisation process. Following the MOO, Compromised Programming (CP), a MCDA technique, is used to assess the solutions according to user defined weighting of various criteria spanning a range of design aspects including technical, environmental, social and economic considerations. The user is then presented with a list of solutions ranked in accordance to the distance each solution is from the ideal or utopian solution. The ESP (described further in Section 2.3), was created as an add-in to WiSDOM, using the results from the tool depending on the scenario or context defined by the user. For the purpose of this study, the MOO objectives, parameters and MCDA criteria weight settings were set to their relevant default settings [18]. It should be noted that although separate locations were chosen for each scenario, two assumptions were made regarding the inputting factors to ensure that the results focused on the removal of EPs:

1) The genetic algorithm objectives, parameters and MCDA criteria weight were set to their default settings. Scenario 2(A) was set to the rural default settings and the other scenarios were set to the urban default settings.

2) The water quality parameters for conventional pollutants inputted into the tool were the same for each location.

More details on WiSDOM can be found in Sadr et al. (2018) [18].

\subsection{Excel Spreadsheet Program}

The ESP which was used to determine the removal of EPs from different treatment unit processes was created from three different separate worksheets. Removal efficiencies were researched for each treatment option taken from the WiSDOM tool. The ESP considered a range of assumptions to allow for a more complete dataset of removal rates for different treatment processes. The different worksheets, 
Emerging Pollutants in Developing Countries: Optimising Sustainable Treatment ... Z. Visanji et al.

explained further in Section 2.3.1, were combined using functions and formulas to allow for a userfriendly spreadsheet program.

\subsubsection{Worksheets and Datasets}

A database was created containing over 500 recorded EPs with data present from many countries. The database was used to gather information on EPs, however only 13 were chosen for the final study as discussed in Section 2.1. The database included abbreviations of EPs, their chemical abstract service (CAS) number, and recorded minimum and maximum concentrations from surface water, groundwater, untreated wastewater, drinking water and treated wastewater.

Spreadsheet A: Consisted of a list of the different treatment unit processes used within the WiSDOM tool. Each of the 42 unit processes [18], [19] were assigned an ID number to allow for transfer of information across spreadsheets. Spreadsheet B: Contained information on the chosen 13 EPs copied over from the database including initial concentrations of the EP which were used for the ESP. Spreadsheet C: Listed the 42 treatment unit processes with the same ID number as spreadsheet A. The minimum and maximum removal rates for each of the $13 \mathrm{EPs}$ were stated as a percentage value. The removal rate of each emerging pollutant equates to the percentage of the chemical which was removed during a treatment process or stage.

Research currently published [5], [17], [20] focuses on the overall removal rate of EPs through different treatment trains and does not focus on individual treatment unit processes. Therefore, information surrounding the breakdown of the removal efficiencies within the effluent at different stages of treatment is unavailable. In addition, insufficient data exists for each EP and each unit process which has been chosen. Consequently, where no data was found for an individual treatment process a removal rate of $0 \%$ was inputted into the cells to produce a complete dataset; allowing for the calculations within the ESP to effectively run. The treatment options set to 0\% removal were: Bar Screen, Grit Chamber, Coarse Screen, Fine Screen, Actiflo, Enhanced Biological Phosphorus Removal (EBPR), PPrecipitation and Soil Aquifer Treatment.

\subsubsection{Creation of the Excel Spreadsheet Program}

To produce the final ESP, different components and datasets were pulled from other tables/spreadsheets (Section 2.3.1) and populated into a singular user-friendly spreadsheet program. The names of the EPs from Spreadsheet B along with a minimum and maximum initial concentration values $(\mathrm{ng} / \mathrm{L})$ were inputted into the first four columns. The top three rows consisted of the stage of treatment, including drop down options allowing the user to select the ID number for a unit process (from Spreadsheet A). The ESP matches the treatment unit processes used within WiSDOM, therefore the ESP contained the same stages and unit processes ID numbers. The drop-down option for the unit processes was taken from Spreadsheet A, as the ID number is changed the treatment name changes to the corresponding process from Spreadsheet C. This allows the ESP to take the relevant removal percentage from Spreadsheet $\mathrm{C}$ of each individual EP, depending on the treatment process chosen. The main section of the ESP involved an equation (Equation 1) which calculated the removal of EPs throughout different unit processes which have been selected. The equation (Equation 1) was used across the ESP which takes the removal rates associated to a unit process from Spreadsheet $\mathrm{C}$ and calculates the new concentration $(\mathrm{ng} / \mathrm{L})$ after that treatment unit. If the concentration after a treatment stage reaches the desired level (defined by the tool user) then the words 'No Further Treatment' will appear, showing the end user where the EP was fully removed. The inbuilt 'IF' function in Excel is used to change the 
information regarding removal rates (from Spreadsheet C), depending on the unit process ID number selected by the user within the drop-down options on the ESP.

$$
Y_{m}=I_{m} \times \prod_{k=1}^{S}\left(1-R_{m, u, k}\right)
$$

Where, m: Contaminant ID; k: Stage of treatment; S: Maximum number of stages considered in the proposed treatment train; $\mathrm{I}_{\mathrm{m}}$ : Influent quality with respect to concentration of $\mathrm{m} ; \mathrm{Y}_{\mathrm{m}}$ : Effluent quality with respect to concentration of $\mathrm{m}$; and $\mathrm{R}_{\mathrm{m}, \mathrm{u}, \mathrm{k}}$ : Contaminant removal rate of the unit process $\mathrm{u}$ in treatment stage $\mathrm{k}$.

An example demonstrating the components of the ESP and Equation (1) are demonstrated below for a treatment train with four treatment stages (see Figure 1) for removal of Diclofenac (DCF). As shown in Figure 1, in the first stage a grit chamber process is selected (used as a preliminary/primary treatment) but does not remove any of the DCF in the wastewater. A Membrane Bioreactor (MBR) was applied in the second stage; this unit process can remove 40 per cent of the DCF. The MBR is followed by a Nanofiltration (NF) and an Ultraviolet (UV) process with DCF removal rates of 60 and 40 percent, respectively. The concentration of DCF after going through all the four stages is $1370 \mathrm{ng} / \mathrm{l}$ which means that the total DCF removal efficiency of this treatment train is 85 per cent.

$$
\begin{gathered}
Y_{D C F}=I_{m} \times \prod_{k=1}^{S=4} R_{m, u, k} \\
Y_{D C F}=9520 \frac{n g}{l} \times[(1-0) \times(1-0.6) \times(1-0.4) \times(1-0.4)] \rightarrow Y_{m}=1370 \frac{\mathrm{ng}}{l}
\end{gathered}
$$

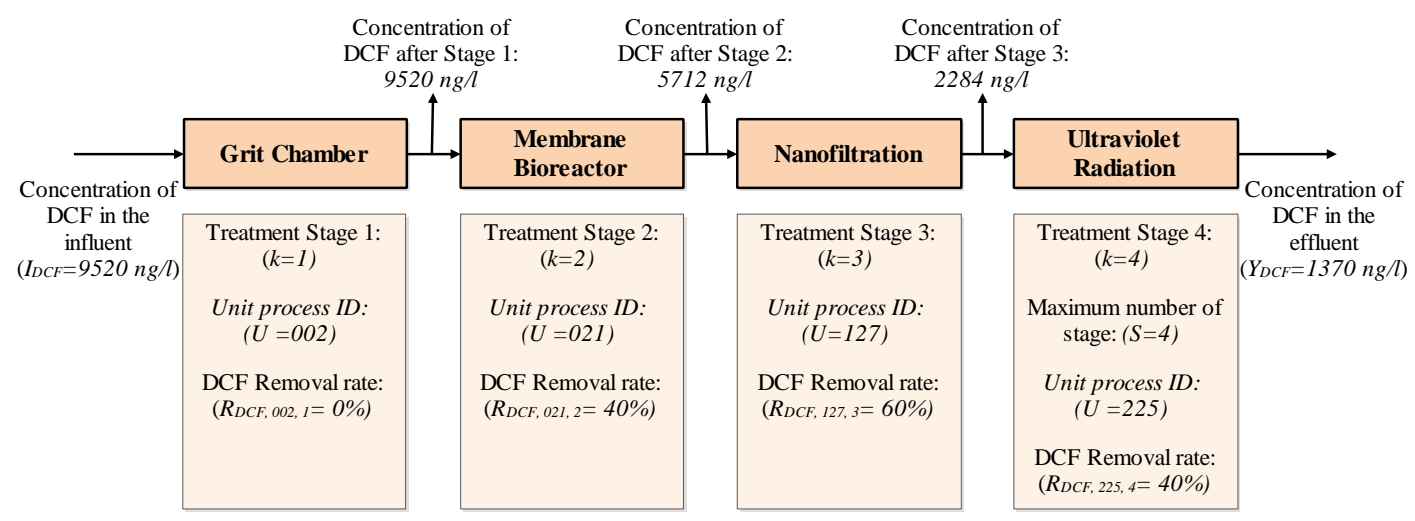

Figure 1: Represents a treatment train schematic showing the input concentration and output concentration of Diclofenac. The percentage values represent the removal rates for the unit process which has been used.

The result from the ESP are displayed in tabular and graphical format. A bar chart of the EPs final concentration in the effluent for both minimum and maximum removal rates is displayed. Results are shown for the final concentration after each treatment train and at the end of each individual unit process. 


\section{Results}

The aim of this study was to analyse the performance of different treatment technologies to determine their efficiency at removing EPs specifically within developing countries. The results for the optimisation of the treatment solutions from the WiSDOM tool were displayed as radar (spider web) charts and the removal of EPs calculated from the ESP were presented as bar charts. The below example displays the results obtained from Scenario 1 which investigated suitable treatment technologies which were able to remove EPs from areas effected by tourism at different scales such as (A) "Occasional Events" and (B) tourism locations. In both sub-scenarios, it was expected that high levels of PCPs and pharmaceuticals would be found in the locations chosen.mostly for your information since the best way to produce a conforming document is by modifying this guide.

\subsection{Scenario 1(A): Determining treatment options for areas that consist of 'Occasional Events' such as Diwali and Ganesh Chaturthi}

Using the results from the WiSDOM tool, it is possible to determine the treatment train solution which is best suited to removing conventional pollutants from 'Occasional Events' in India. Figure 2, shows that the treatment train suited to removing most of the conventional pollutants was solution S4986 (Coarse Screen - Actiflo - Membrane Bioreactor - Soil Aquifer Treatment - Chlorine Gas). This solution performed highly, demonstrating low quantities of phosphorus, COD and turbidity. There were high levels of total nitrogen remaining in the effluent compared to solution S4328 (Grit Chamber - Actiflo- Low Loaded Activated Sludge with de-nitrification and Secondary Sedimentation - Soil Aquifer Treatment - Chlorine Gas) however, S4328 scored poorly at removing COD, turbidity, suspended solids and phosphorus. In Figure 2, solutions S4986 and S4328 are presented, both required high labour and CAPEX. Although S4986 required more energy than S4328, the first solution improved the quality of the effluent water to better meet the standards of India. However, when looking at the removal of EPs during this treatment train, Figure 3E, shows a low removal rate of EPs, with some not removed at all. The solution which demonstrated a higher removal rate for all EPs was S4707 (Coarse Screen - Sedimentation without Coagulant - Membrane Bioreactor - Soil Aquifer Treatment - Chlorine Gas). Solution S4707 was able to remove BPA, CIP, DCF, DMP, NPX, NP, NOR and TCS at a removal rate greater than $90 \%$. 

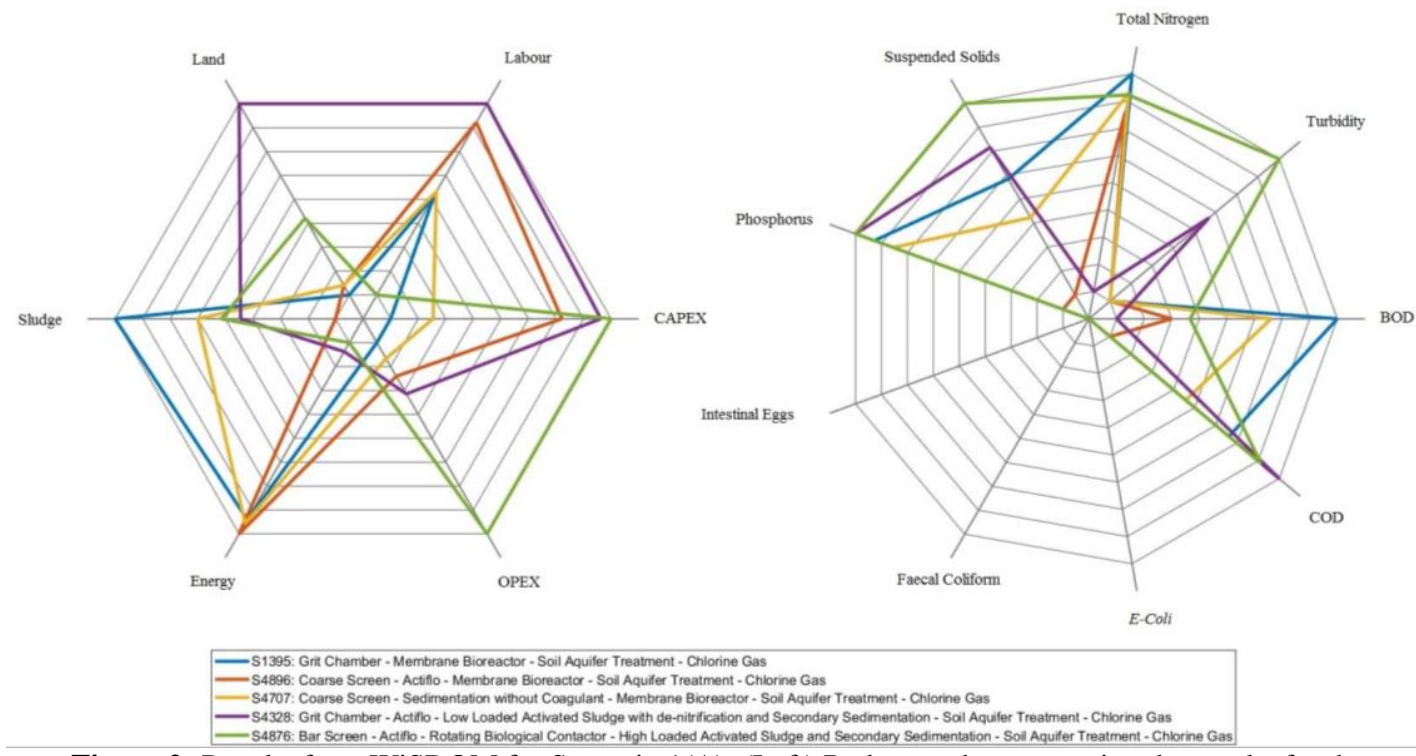

Figure 2: Results from WiSDOM for Scenario 1(A): (Left) Radar graph representing the results for the performance of different sustainability indicator objectives; (Right) Radar graph representing the results for the performance of the removal of conventional pollutants. E-Coli, Faecal Coliform and Intestinal Eggs were fully removed during all treatment solutions.
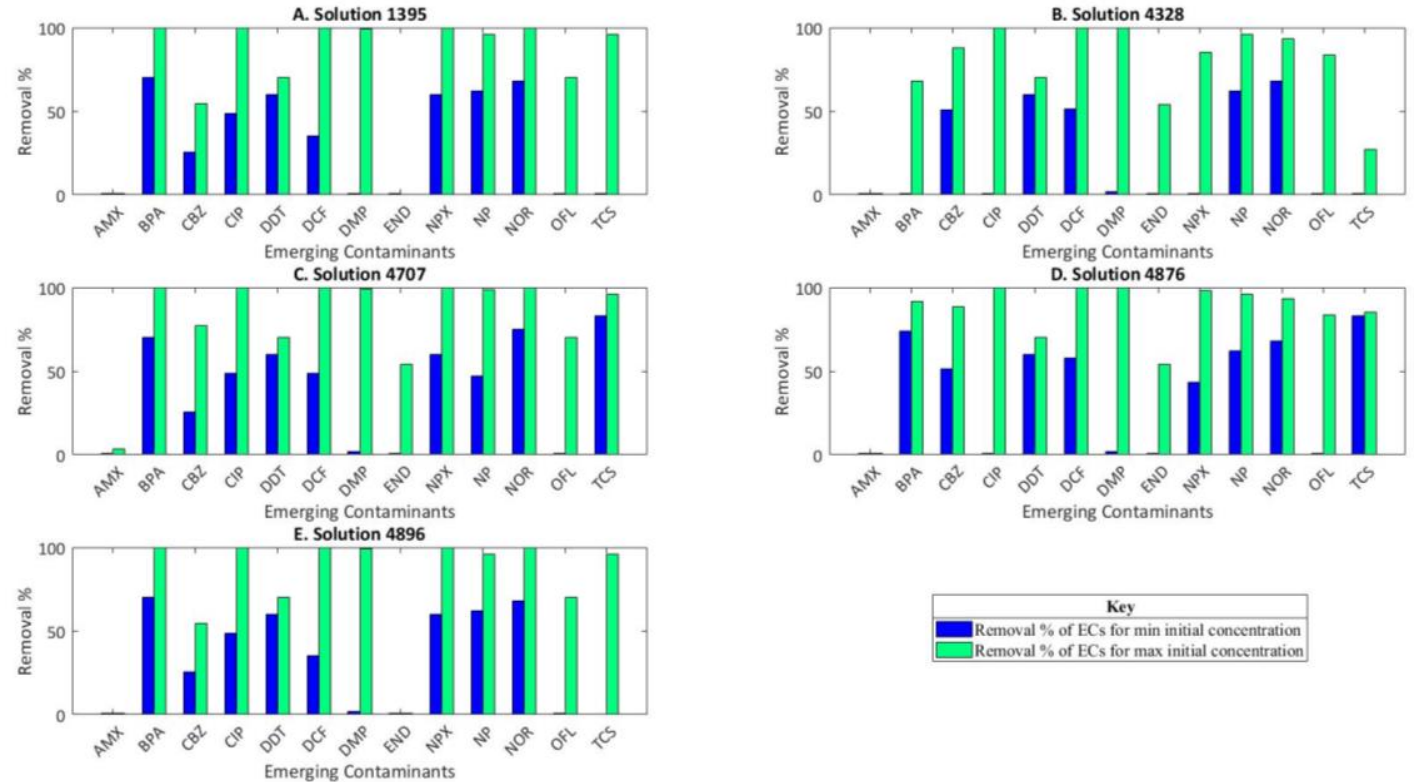

Figure 2: Percentage of EPs removed for the treatment options produced by the WiSDOM tool

(Figure 2) for Scenario 1(A). 


\subsection{Scenario 1(B): Determining treatment options for areas in India such as 'The Golden Triangle', with known high tourism levels}

Figure 4, shows that the WiSDOM tool found solution S9823 (Grit Chamber - Actiflo - Membrane Bioreactor - Soil Aquifer Treatment - Chlorine Gas) performed highly in regard to OPEX and labour, however the other sustainability indicators were outperformed by other solutions. Solutions S9892 (Bar Screen - Sedimentation without Coagulant - Low Loaded Activated Sludge + Secondary Sedimentation - Soil Aquifer Treatment - Chlorine Gas) and S9826 (Grit Chamber - Dissolved Air Flotation (DAF) with Coagulant - Trickling Filter + Secondary Sedimentation - Soil Aquifer Treatment and Chlorine Gas) exhibited similar results with the later containing higher energy requirements. Figure 4, represents that S9777 (Grit Chamber - Actiflo - Submerged Aerated Filter - Soil Aquifer Treatment and Chlorine Gas) was outperformed by the other solutions, as this treatment option was unable to remove suspended solids, total nitrogen, turbidity, BOD and COD from the effluent to the high level of the other solutions. Although S9823 scored low regarding total nitrogen and BOD, this solution was able to remove the other conventional pollutants from the effluent. When examining the removal of EPs from the different treatment solutions in Figure 5A, solution S9777 was ineffective at removing END, NPX and TCS from the effluent. Both S9823 (Figure 5B) and S9826 (Figure 5C) were unable to remove AMX and END. However, S9892 and S9877 were able to remove all the thirteen EPs, with S9877 removing a higher percentage of AMX but a lower amount of TCS (Figure 5D) and S9877 removing a higher level of TCS in comparison to AMX (Figure 5E). Comparing these results to the outcome of the WiSDOM tool (Figure 4) shows that S9877 performed worst in the sustainability indicator objectives. Figure 4, demonstrates that S9877 out performs S9892 when removing phosphorus only. Therefore, S9892 is the best treatment solution which meets all criteria and can effectively remove both conventional pollutants and EPs.

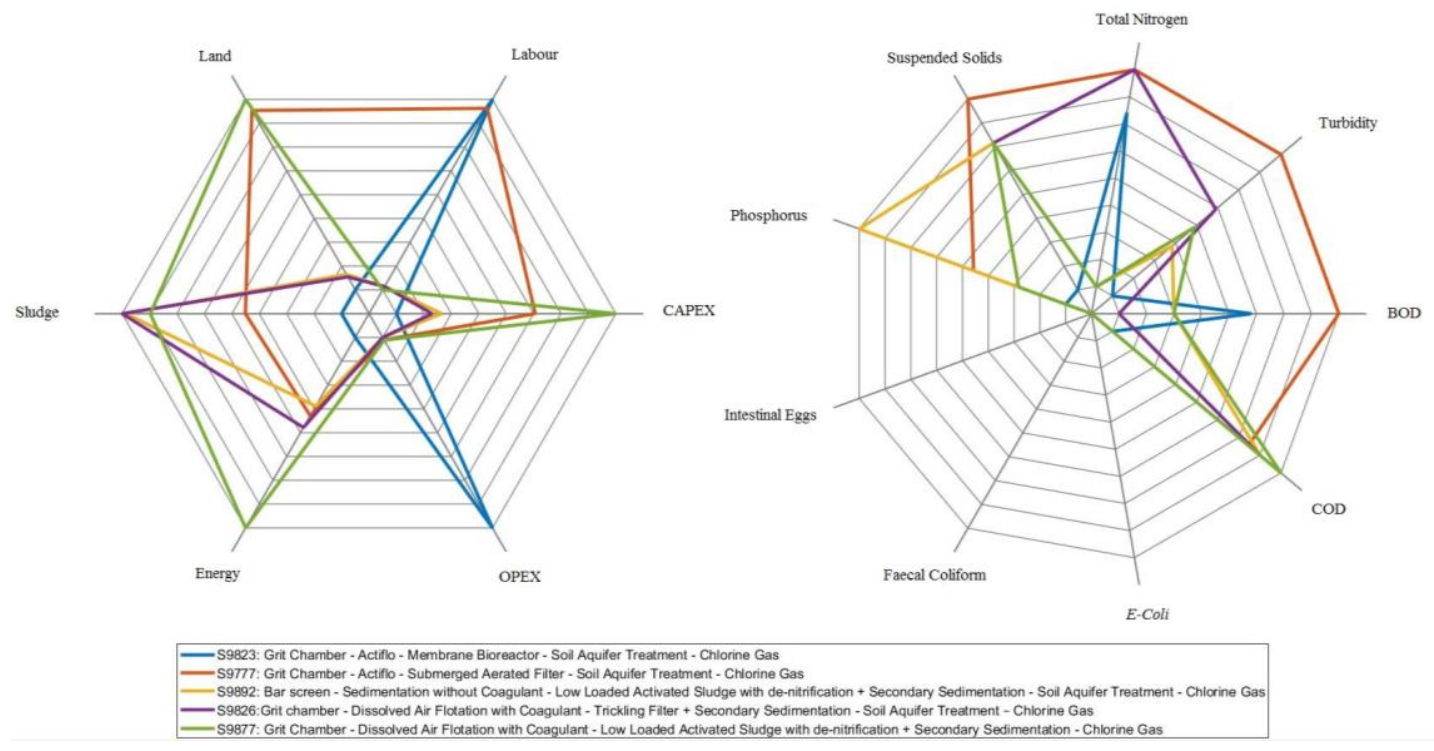

Figure 4: Results from WiSDOM for Scenario 1(B): (Left) Radar graph representing the results for the performance of different sustainability indicator objectives; (Right) Radar graph representing the results for the performance of the removal of conventional pollutants. E-Coli, Faecal Coliform and Intestinal Eggs were fully removed during all treatment solutions. 

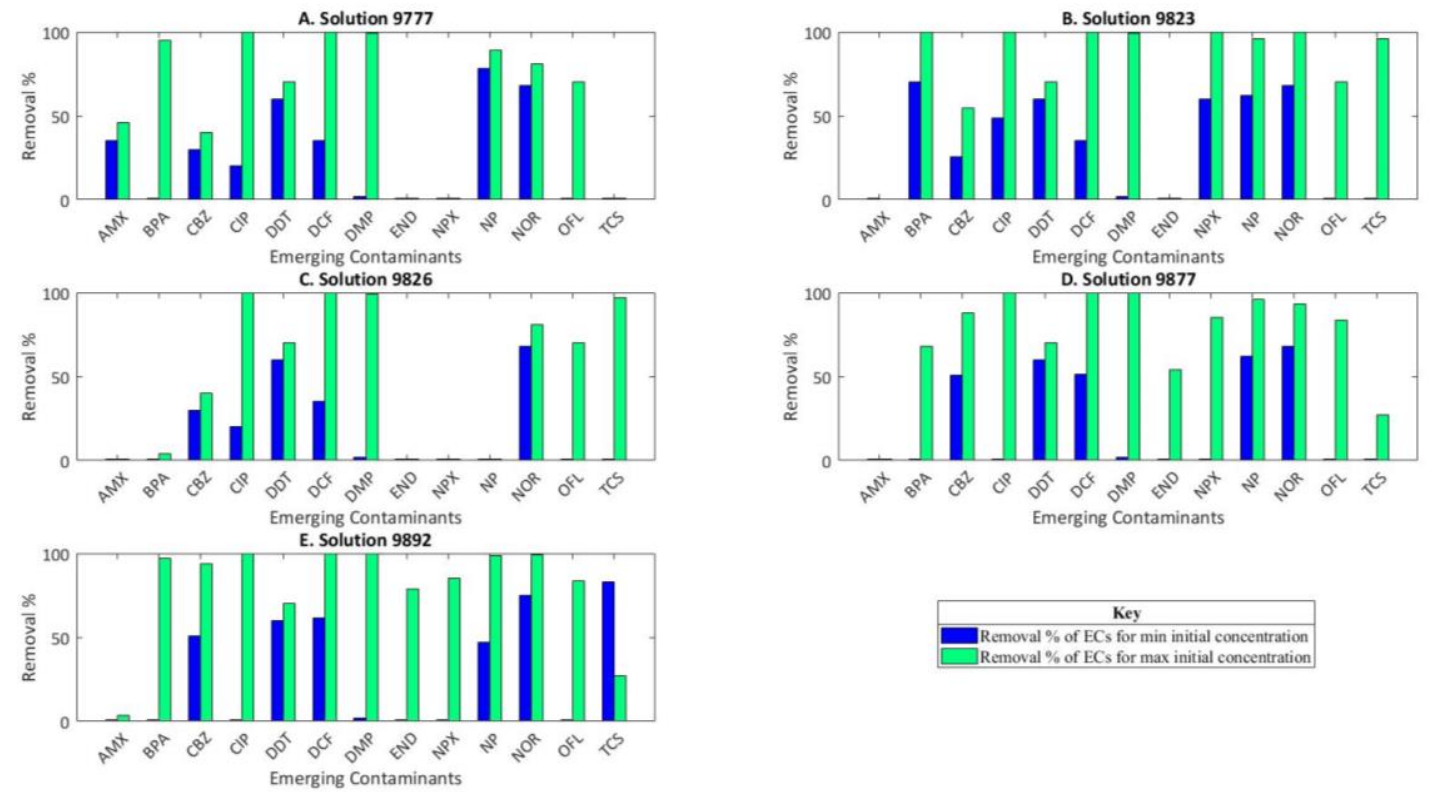

Figure 5: Percentage of EPs removed for the treatment options produced by the WiSDOM tool

(Figure 4) for Scenario 1(B).

The example solutions presented above for both Scenario 1(A) and 1(B) are intended to demonstrate the functionalities of the developed tool and response to the user inputs. Sanity of the tool provided solutions is yet to be further tested and heavily depends on the input data quality.

\section{Conclusions}

The overall aim of this study was to analyse sustainable treatment options for the removal of EPs within developing countries; India was used as a case study. An add-on ESP was created for WiSDOM which can also be used as a stand-alone application to provide information regarding the removal of EPs. The study displays the results of treatment train solutions which are suited at removing EPs from areas affected by high levels of tourism. Scenario 1(A) looked at areas that consist of 'Occasional Events' and the treatment option suited to removing EPs consisted of: Coarse Screen - Sedimentation without Coagulant - Membrane Bioreactor - Soil Aquifer Treatment - Chlorine Gas. Scenario 1(B) looked at a specific tourism location and found that the technologies suited to removing EPs used: Bar Screen Sedimentation without Coagulant - Low Loaded Activated Sludge + Secondary Sedimentation - Soil Aquifer Treatment - Chlorine Gas. This study has provided the basis for further research concerning the removal of EPs. The main limitation with this research occurred due to the lack of data regarding the removal of EPs from different treatment trains. Advances are needed in regard to funding and access to equipment within India to allow for further investigations to fill the current gap within the literature. Primary data collection would allow for more accurate removal rates during different treatment stages. To further this work, the functionality of the add-on worksheet can be integrated directly into WiSDOM by expanding the source code. Additionally, the EP removal model can be incorporated into the MOO process within WiSDOM by imposing further water quality constraints on the search. 


\section{Acknowledgements}

This work was supported by the EPSRC in the UK via grant EP/R512254/1 awarded for the Water Informatics, Science and Engineering (WISE) centre for doctoral training. Part of this study describes work being carried out under EC FP7-funded projects SARASWATI (Grant agreement no: 308672) and NERC project on the Fate and management of Emerging Contaminants (Grant No. NE/R003548/1). The project sponsors were not involved in the study design, collection, analysis and interpretation of data or in the writing of the manuscript.

\section{References}

[1] J. M. Philip, U. K. Aravind, and C. T. Aravindakumar, "Emerging contaminants in Indian environmental matrices - A review," Chemosphere, vol. 190, no. Supplement C, pp. 307-326, Jan. 2018.

[2] D. Montes-Grajales, M. Fennix-Agudelo, and W. Miranda-Castro, "Occurrence of personal care products as emerging chemicals of concern in water resources: A review," Sci. Total Environ., vol. 595, pp. 601-614, Oct. 2017.

[3] M. Picot Groz, M. J Martinez Beuno, D. Rosain, H. Fenet, C. Casellas, C. Pereira, V. Maria, M.J. Bebianno, and E. Gomez, "Detection of emerging contaminants (UV filters, UV stabilizers and musks) in marine mussels from Portuguese coast by QuEChERS extraction and GC-MS/MS," Sci. Total Environ., vol. 493, pp. 162-169, Sep. 2014.

[4] X. Li, W. Zheng, and W. R. Kelly, "Occurrence and removal of pharmaceutical and hormone contaminants in rural wastewater treatment lagoons," Sci. Total Environ., vol. 445-446, pp. 22-28, Feb. 2013.

[5] Y. Luo, W. Guo, H. H. Ngo, F. I. Hai, J. Zhang, S. Liang, and X. C. Wang, "A review on the occurrence of micropollutants in the aquatic environment and their fate and removal during wastewater treatment," Sci. Total Environ., vol. 473-474, pp. 619-641, Mar. 2014.

[6] A. Nikolaou, S. Meric, and D. Fatta, "Occurrence patterns of pharmaceuticals in water and wastewater environments," Anal. Bioanal. Chem., vol. 387, no. 4, pp. 1225-1234, Feb. 2007.

[7] A. Jurado, E. Vàzquez-Suñé, J. Carrera, M. López de Alda, E. Pujades, and D. Barceló, "Emerging organic contaminants in groundwater in Spain: A review of sources, recent occurrence and fate in a European context," Sci. Total Environ., vol. 440, pp. 82-94, Dec. 2012.

[8] E. Stumm-Zollinger and G. M. Fair, "Biodegradation of Steroid Hormones," J. Water Pollut. Control Fed., vol. 37, no. 11, pp. 1506-1510, 1965.

[9] N. H. Hashim, M. H. Nasir, and M. S. Ramlee, "Emerging Pollutant of Concen: Occurrence of Pharmaceutical Compounds in Asia with Particular Prefernce to Southeast Asia Countries,"

MATEC Web Conf., vol. 47, no. 05026, 2016. 
Emerging Pollutants in Developing Countries: Optimising Sustainable Treatment ... Z. Visanji et al.

[10] T. Deblonde, C. Cossu-Leguille, and P. Hartemann, "Emerging pollutants in wastewater: A review of the literature," Int. J. Hyg. Environ. Health, vol. 214, no. 6, pp. 442-448, Nov. 2011.

[11] N. Chaukura, W. Gwenzi, N. Tavengwa, and M. M. Manyuchi, "Biosorbents for the removal of synthetic organics and emerging pollutants: Opportunities and challenges for developing countries," Environ. Dev., vol. 19, pp. 84-89, Jul. 2016.

[12] A. E. A. A. Said, A. A. M. Aly, M. M. A El-Whab, S. A. Soliman, A. A. A El-Hafez, V. Helmey, and M. N. Goda, "Application of Modified Bagasse as a Biosorbent for Reactive Dyes Removal from Industrial Wastewater," J. Water Resour. Prot., vol. 5, pp. 10-17, Jul. 2013.

[13] D. S. Zandaryaa and D. Frank-Kamenetsky, "Emerging Pollutants in Water and Wastewater: UNESCO-HELCOM Case Study on Pharmaceuticals in the Aquatic and Marine Environment in the Baltic Sea Region,” 2015.

[14] R. Mailler, J. Gasperi, Y. Coquet, A. Buleté, E. Vulliet, S. Deshayes, S. Zedek, C. MirandeBret, V. Eudes, A. Bressy, E. Caupos, R. Moilleron, G. Chebbo and V. Rocher, "Removal of a wide range of emerging pollutants from wastewater treatment plant discharges by micro-grain activated carbon in fluidized bed as tertiary treatment at large pilot scale," Sci. Total Environ., vol. 542, Part A, pp. 983-996, Jan. 2016.

[15] K. M. Gani and A. A. Kazmi, "Contamination of Emerging Contaminants in Indian Aquatic Sources: First Overview of the Situation,” J. Hazard. Toxic Radioact. Waste, vol. 21, no. 3, Jul. 2017.

[16] K. C. Machado, M. T. Grassi, C. Vidal, I. C. Pescara, W. F. Jardim, A. N. Fernandes, F. F. Sodré, F. V. Almedia, J. S Santana, M. C. Canela, C. R. O. Nunes, K. M. Bichinho, F. J. R. Severo "A preliminary nationwide survey of the presence of emerging contaminants in drinking and source waters in Brazil," Sci. Total Environ., vol. 572, pp. 138-146, Dec. 2016.

[17] B. Petrie, R. Barden, and B. Kasprzyk-Hordern, "A review on emerging contaminants in wastewaters and the environment: Current knowledge, understudied areas and recommendations for future monitoring," Water Res., vol. 72, pp. 3-27, Apr. 2015.

[18] S. M. K. Sadr, M. B. Johns, F. A. Memon, M. Morley, and D. Savić, "Development and Application of a User-Friendly Decision Support Tool for Optimization of Wastewater Treatment Technologies in India," presented at the HIC 2018: 13th International Conference on Hydroinformatics, Palermo, Italy, 2018.

[19] D. Joksimovic, "Decision Support System for Planning of integrated Water Reuse Projects," Jan. 2007.

[20] N. H. Tran, M. Reinhard, and K. Y.-H. Gin, "Occurrence and fate of emerging contaminants in municipal wastewater treatment plants from different geographical regions-a review," Water Res., vol. 133, pp. 182-207, Apr. 2018. 